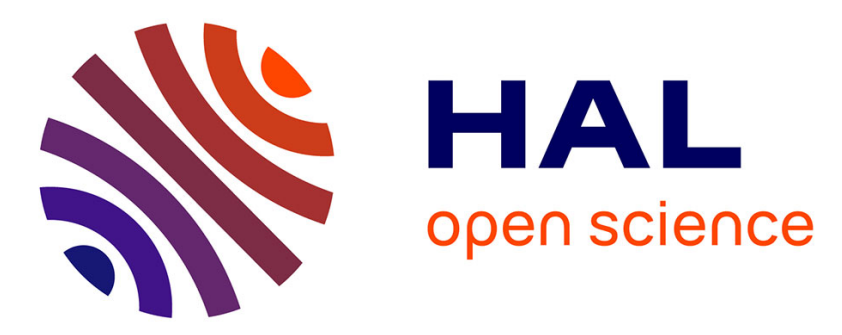

\title{
Zonotopic guaranteed state estimation for uncertain systems
}

Vu Tuan Hieu Le, Cristina Stoica, T. Alamo, E.F. Camacho, Didier Dumur

\section{To cite this version:}

Vu Tuan Hieu Le, Cristina Stoica, T. Alamo, E.F. Camacho, Didier Dumur. Zonotopic guaranteed state estimation for uncertain systems. Automatica, 2013, 49 (11), pp.3418-3424. 10.1016/j.automatica.2013.08.014 . hal-00876455

\section{HAL Id: hal-00876455 \\ https://hal-centralesupelec.archives-ouvertes.fr/hal-00876455}

Submitted on 16 Mar 2020

HAL is a multi-disciplinary open access archive for the deposit and dissemination of scientific research documents, whether they are published or not. The documents may come from teaching and research institutions in France or abroad, or from public or private research centers.
L'archive ouverte pluridisciplinaire HAL, est destinée au dépôt et à la diffusion de documents scientifiques de niveau recherche, publiés ou non, émanant des établissements d'enseignement et de recherche français ou étrangers, des laboratoires publics ou privés. 


\title{
Zonotopic guaranteed state estimation for uncertain systems
}

\author{
V.T.H. Le ${ }^{\mathrm{a}}$, C. Stoica ${ }^{\mathrm{a}, *}$, T. Alamo ${ }^{\mathrm{b}}$, E.F. Camacho ${ }^{\mathrm{b}}$, D. Dumur ${ }^{\mathrm{a}}$ \\ ${ }^{a}$ SUPELEC Systems Sciences (E3S) - Automatic Control Department, 3 rue Joliot Curie, F-91190 Gif-sur-Yvette cedex, France \\ (e-mail: tuanhieu_bk@yahoo.fr, cristina.stoica@supelec.fr,didier.dumur@supelec.fr) \\ ${ }^{b}$ Department of Ingeniería de Sistemas y Automática, Universidad de Sevilla, Camino de los Descubrimientos, 41092 Sevilla, Spain \\ (e-mail: alamo@cartuja.us.es,eduardo@esi.us.es)
}

\begin{abstract}
This paper presents a new approach for guaranteed state estimation based on zonotopes for linear discrete time multivariable systems with interval multiplicative uncertainties, in the presence of bounded state perturbations and noises. At each sample time, the presented approach computes a zonotope which contains the real system state. A $P$-radius based criterion is minimized in order to decrease the size of the zonotope at each sample time and to obtain a more and more accurate state estimation. The proposed approach allows to efficiently handle the trade-off between the complexity of the computation and the accuracy of the estimation. An illustrative example is analyzed in order to highlight the advantages of the proposed state estimation technique.
\end{abstract}

Keywords: set-membership estimation, interval uncertainty, multivariable systems, zonotope, Linear Matrix Inequality.

\section{Introduction}

The state estimation is of great interest for feedback control and diagnosis of dynamical systems. This problem can be formulated as follows: given a mathematical model of a real system and allowing some state perturbations and noise corrupted measurements, the state of the real system has to be estimated. In the literature, the state estimation problem is mainly treated using stochastic approach or deterministic approach.

Stochastic procedures (e.g., the Kalman filter Kalman (1960); Maybeck (1979); Sorenson (1983)) have been developed since 1960s and are still a widely applied technique. These approaches are based on probabilistic assumptions on perturbations and noise. The state estimation is done by minimizing the variance of the state estimation error. However, these probabilistic assumptions are sometimes not realistic and difficult to validate (e.g., in a real application, it is not easy to know the distribution law of perturbations).

Deterministic approach or set-membership estimation assumes that the perturbations and the noises are unknown but bounded (Schweppe (1968); Kurzhanski \& Vályi (1996); Walter \& Piet-Lahanier (1989); Vicino \& Zappa (1996); Combastel (2003); Alamo et al. (2005)). Under this hypothesis, the information about the system states at each sample time is characterized as a compact set containing all possible system states that are consistent with the measurement sample, the perturbations, the uncertainties, and the noise. No other hypotheses related to the distribution of perturbations and noises are necessary.

\footnotetext{
Part of the material in this paper was presented at the 18th IFAC World Congress 2011, Milan.

*Corresponding author. Tel.: +331698513 78; Fax: +33169851389. E-mail address cristina.stoica@ supelec.fr (C. Stoica).
}

In the set-membership approach different domain representations can be used to bound the consistent set, such as polytopes (boxes, parallelotopes) (Walter \& Piet-Lahanier (1989); Vicino \& Zappa (1996)), ellipsoids (Schweppe (1968); Witsenhausen (1968); Bertsekas \& Rhodes (1971), Chernous'ko (1994); Milanese et al. (1996); Kurzhanski \& Vályi (1996); Durieu et al. (2001); Polyak et al. (2004)), zonotopes (Puig et al. (2001); Combastel (2003); Alamo et al. (2005)). When different domain representations are used, there is a trade-off between the computation load and the precision of the estimation. On the one hand, due to the simplicity of the formulation, ellipsoids have been used by different authors (Kurzhanski \& Vályi (1996); Durieu et al. (2001)). On the other hand, polytopes (or certain classes of polytopes, e.g., parallelotopes) are proposed to obtain a better estimation accuracy (Vicino \& Zappa (1996), Walter \& Piet-Lahanier (1989)). They can be used for an exact representation of the variation domains of the system's state in a linear formulation. However, efficient results can be obtained only for a reasonable number of vertices of polytopes (Walter \& Piet-Lahanier (1989)).

In recent years, zonotopes (Vicino \& Zappa (1996); Kühn (1998); Combastel (2003); Alamo et al. (2005); Althoff et al. (2007)) have received increased attention because of their accuracy and compactness of representation compared to ellipsoids and their reduced complexity compared to polytopes. Contrary to ellipsoids, the Minkowski sum of two zonotopes is a zonotope, this property being very useful in set-membership estimation. Moreover, zonotopes can represent uncertainties due to perturbations independently in each direction of the statespace. In addition zonotopes are a suitable representation for controlling the wrapping effect (Kühn (1998)) (the growth of the domain representation due to uncertainty at each sample time). The zonotopic domain is used for many applications: 
reachability analysis (Althoff et al. (2007)), collision detection (Guibas et al. (2005)), state estimation (Combastel (2003); Alamo et al. (2005); Puig et al. (2001)), ultimate bound (Stoican et al. (2011)), and fault diagnosis (Combastel et al. (2008)).

In Puig et al. (2001) the measured output is utilized to estimate the state by means of a gain matrix. In Combastel (2003) a singular value decomposition is used to obtain an outer approximation of the intersection between the uncertain trajectory and the region of the state-space that is consistent with the measured output vector. In Alamo et al. (2005), interval arithmetics and zonotopic sets are used to obtain a guaranteed state estimation for single-output systems with interval parameter uncertainties. The solution is elaborated online as a family of zonotopes parameterized by a free vector. Two different criteria are used to minimize the size of this zonotope. The segment minimization offers a fast computation of the optimal parameterizing vector but the results can be conservative. The volume minimization offers a better result by solving a convex optimization problem on each iteration, sometimes leading to a very narrow zonotope, i.e., the uncertainty in one direction can remain extremely large, but at the same time the volume of the zonotope tends to zero.

Most of the works cited above solve the estimation problem when the plant model is known and the uncertainty is only related to state perturbations and measurement noises. In Polyak et al. (2004), a conservative assumption on the relation between the state uncertainty matrix and the state perturbation is used to obtain an ellipsoidal state estimation for multi-output systems.

Based on the results in Alamo et al. (2005), one contribution of this paper is to present a new optimization criterion that minimizes the $P$-radius associated to the zonotope which is an original notion to characterize the size of the zonotope in order to obtain good accuracy and reasonable computation load. Our method allows performing an off-line optimization which is a major advantage for real-time applications. The proposed method offers a trade-off between the segment and the volume minimization methods (Alamo et al. (2005)). Initially developed in the case of linear discrete-time single-output systems with bounded state perturbations and measurement noise, part of this method was published in Le et al. (2011). An extension for single-output uncertain systems has been proposed in Le et al. (2012). An original contribution of the present paper is the generalization of this result to multi-output systems with interval multiplicative uncertainties, bounded state perturbations and measurement noises. A first idea is to consider the multioutput system as separate single-output systems. A second idea is to estimate the system's state based on the information from all the output sensors at the same time in order to obtain a better accuracy compared to the first idea.

The paper is organized as follows. Section 2 presents useful mathematical notations and basic definitions. In Section 3, the class of dynamical systems used along this paper is defined. The next section formulates the main results of this paper presenting a new approach to compute an outer bound of the state estimation by zonotopes for multi-output systems with interval uncertainties. In Section 5, an example is proposed in order to illustrate the advantages of the developed methods. Finally, some concluding remarks and future works are discussed.

\section{Notations, Basic Definitions and Properties}

An interval $[a, b]$ is defined as the set $\{x: a \leq x \leq b\}$, with $\operatorname{mid}[a, b]=\frac{a+b}{2}$ and $\operatorname{rad}[a, b]=\frac{b-a}{2}$ denoting its center and its radius, resp. The unitary interval is $\mathbf{B}=[-1,1]$. The set of real compact intervals $[a, b]$, where $a, b \in \mathbb{R}$ and $a \leq b$ is denoted by $\mathbb{I}$. A box $\left(\left[a_{1}, b_{1}\right], \ldots,\left[a_{n}, b_{n}\right]\right)^{\top}$ is an interval vector. A unitary box in $\mathbb{R}^{n}$ denoted by $\mathbf{B}^{n}$ is a box composed by $n$ unitary intervals. An interval matrix $[M] \in \mathbb{I}^{n \times m}$ is a matrix whose elements are intervals. It means that each element $M_{i j}$, with $i=1, \ldots, n, j=1, \ldots, m$ of this matrix is defined as the set $M_{i j}=\left\{m_{i j}: a_{i j} \leq m_{i j} \leq b_{i j}\right\}$. In the matrix space, the interval matrix is a hyperrectangle and, hence, a convex set. Let $\operatorname{vert}[M]$ denote the set of all matrices $G=\left[g_{i j}\right], i=1, \ldots, n$, $j=1, \ldots, m$, such that $g_{i j}=a_{i j}$ or $g_{i j}=b_{i j}$. The notations $\operatorname{mid}[M]_{i j}=\frac{a_{i j}+b_{i j}}{2}$ and $\operatorname{rad}[M]_{i j}=\frac{b_{i j}-a_{i j}}{2}$ define the center and the radius of an interval matrix $[M]$, resp. The row sum diagonal matrix of a matrix $M \in \mathbb{R}^{n \times m}$ (Combastel (2003)) is defined as $r s(M)=\operatorname{diag}\left(\left[\ldots, \tilde{m}_{i i}, \ldots\right]\right)$, with $\tilde{m}_{i i}=\sum_{j=1}^{m}\left|m_{i j}\right|, i=1, \ldots, n$.

The Minkowski sum of two sets $X$ and $Y$ is defined by $X \oplus Y=\{x+y: x \in X, y \in Y\}$. A strip $S$ is defined as the set $\left\{x \in \mathbb{R}^{n}:\left|c^{\top} x-y\right| \leq \phi\right\}$, with $c \in \mathbb{R}^{n}$ and $y, \phi \in \mathbb{R}$.

Zonotopes are a special class of convex symmetric polytopes. A $m$-zonotope in $\mathbb{R}^{n}$ can be defined as the affine image of a $m$ dimensional hypercube in $\mathbb{R}^{n}$. Given a vector $p \in \mathbb{R}^{n}$ and a matrix $H \in \mathbb{R}^{n \times m}$, a $m$-zonotope $Z$ is the set $Z=p \oplus H \mathbf{B}^{m}=\{p+$ $\left.H x: x \in \mathbf{B}^{m}\right\}$. This is the Minkowski sum of the $m$-segments defined as $m$ columns of matrix $H$ in $\mathbb{R}^{n}$. The $P$-radius of a zonotope $Z=p \oplus H \mathbf{B}^{m}$ is defined as $L=\max _{z \in Z}\|z-p\|_{P}^{2}$, where $P=P^{\top}>0$ is a symmetric and positive definite matrix.

Property 1: The Minkowski sum of two zonotopes $Z_{1}=p_{1} \oplus$ $H_{1} \mathbf{B}^{m_{1}} \in \mathbb{R}^{n}$ and $Z_{2}=p_{2} \oplus H_{2} \mathbf{B}^{m_{2}} \in \mathbb{R}^{n}$ is also a zonotope defined by $Z=Z_{1} \oplus Z_{2}=\left(p_{1}+p_{2}\right) \oplus\left[\begin{array}{ll}H_{1} & H_{2}\end{array}\right] \mathbf{B}^{m_{1}+m_{2}}$.

Property 2: The image of a zonotope $Z=p \oplus H \mathbf{B}^{m} \in \mathbb{R}^{n}$ by a linear mapping $K$ can be computed by a standard matrix product $K Z=(K p) \oplus(K H) \mathbf{B}^{m}$.

Property 3: (Zonotope reduction Combastel (2003); Alamo et al. (2005)) Given the zonotope $Z=p \oplus H \mathbf{B}^{m} \in \mathbb{R}^{n}$ and the integer $s$, with $n<s<m$, denote $\hat{H}$ the resulting matrix after reordering the columns of the matrix $H=\left[h_{1} \ldots h_{i} \ldots h_{m}\right]$ in decreasing order of Euclidean norm $\left(\hat{H}=\left[\hat{h}_{1} \ldots \hat{h}_{i} \ldots \hat{h}_{m}\right]\right.$, with $\left\|\hat{h}_{i}\right\|_{2} \geq$ $\left.\left\|\hat{h}_{i+1}\right\|_{2}\right)$. Denote by $\hat{H}_{T}$ the matrix obtained from the first $s-n$ columns of matrix $\hat{H}$ and by $\hat{H}_{Q}$ the rest of the matrix $\hat{H}$. Then the following inclusion is obtained $Z \subseteq p \oplus\left[\begin{array}{ll}\hat{H}_{T} & r s\left(\hat{H}_{Q}\right)\end{array}\right] \mathbf{B}^{s}$.

Property 4: (Zonotope inclusion Alamo et al. (2005)) Consider a family of zonotopes represented by $Z=p \oplus[M] \mathbf{B}^{m}$, where $p \in \mathbb{R}^{n}$ is a real vector and $[M] \in \mathbb{I}^{n \times m}$ is an interval matrix. A zonotope inclusion is an outer approximation of this family defined by $p \oplus[\operatorname{mid}[M] \quad \operatorname{rs}(\operatorname{rad}[M])] \mathbf{B}^{m+n}$.

Property 5: Given an interval matrix $[M] \in \mathbb{I}^{n \times p}$ and a real matrix $N \in \mathbb{R}^{p \times q}$, the center and the radius of the product $[M] N$ are given by $\operatorname{mid}([M] N)=(\operatorname{mid}[M]) N$ and $\operatorname{rad}([M] N)=$ $(\operatorname{rad}[M])|N|$, where $|N|$ refers to the matrix formed with the absolute value of each element of $N$.

Property 6: (Alamo et al. (2005)). Given a zonotope $Z=$ $p \oplus H \mathbf{B}^{r} \in \mathbb{R}^{n}$, a strip $S=\left\{x \in \mathbb{R}^{n}:\left|c^{\top} x-y\right| \leq \phi\right\}$ and a 
vector $\lambda \in \mathbb{R}^{n}$, define a vector $\hat{p}(\lambda)=p+\lambda\left(y-c^{\top} p\right) \in \mathbb{R}^{n}$ and a matrix $\hat{H}(\lambda)=\left[\begin{array}{ll}\left(I-\lambda c^{\top}\right) H & \phi \lambda\end{array}\right]$. Then a family of zonotopes parameterized by $\lambda$ that contains the intersection of a zonotope and a strip is obtained such as $Z \cap S \subseteq \hat{Z}(\lambda)=\hat{p}(\lambda) \oplus \hat{H}(\lambda) B^{r+1}$.

\section{Problem formulation}

Consider the following linear discrete-time invariant system:

$$
\left\{\begin{array}{l}
x_{k+1}=A x_{k}+\omega_{k} \\
y_{k}=C x_{k}+v_{k}
\end{array}\right.
$$

where $x_{k} \in \mathbb{R}^{n_{x}}$ is the state of the system, $y_{k} \in \mathbb{R}^{n_{y}}$ is the measured output at sample time $k$ and the pair $(C, A) \in \mathbb{R}^{n_{y} \times n_{x}} \times$ $\mathbb{R}^{n_{x} \times n_{x}}$ is detectable (Combastel (2003), Plarre \& Bullo (2008)), with $A$ a constant unknown matrix belonging to an interval matrix $[A]$. The vector $\omega_{k} \in \mathbb{R}^{n_{x}}$ represents the state perturbation and $v_{k} \in \mathbb{R}^{n_{y}}$ is the measurement perturbation (noise, offset, etc.). It is assumed that the perturbations and the initial state are bounded: $\omega_{k} \in W, v_{k} \in V$ and $x_{0} \in X_{0}$, where $W$ and $X_{0}$ are zonotopes and $V$ is a box. To simplify the computation, $V$ and $W$ are assumed to be centered at the origin. Note that if this assumption is not satisfied, a change of coordinates can be used. From the definition of a zero-centered zonotope, $W$ and $V$ can be written as $W=F \mathbf{B}^{n_{\omega}}$ and $V=\Phi \mathbf{B}^{n_{y}}$, with the matrix $F \in \mathbb{R}^{n_{x} \times n_{\omega}}$ and the diagonal matrix $\Phi \in \mathbb{R}^{n_{y} \times n_{y}}$. It is assumed that the interval matrix $[A]$ is quadratically stable (not necessary if $\operatorname{rad}[A]=0$, see Le et al. (2011)) for a common quadratic Lyapunov function. This assumption is not restrictive because in many applications the matrix $A$ is given by a closed-loop matrix $\tilde{A}+\tilde{B} K$, with $\tilde{A}, \tilde{B}$ the open-loop matrices and $\tilde{A} \in[\tilde{A}]$, $\tilde{B} \in[\tilde{B}]$. A feedback gain $K$ can be computed by solving a Linear Matrix Inequality problem (Mao \& Chu (2003); Alamo et al. (2008)), so that this assumption is satisfied.

With these notations, the exact uncertain set and the consistent state set are defined as in Alamo et al. (2005).

Definition 1: Given the system (1) and a measured output vector $y_{k}$, the consistent state set at time $k$ (the state set which is consistent with the measured output vector $y_{k}$ ) is defined as $X_{y_{k}}=\left\{x \in \mathbb{R}^{n}:\left(y_{k}-C x\right) \in V\right\}$.

Definition 2: Consider the system (1). The exact uncertain set $X_{k}=\left(A X_{k-1} \oplus W\right) \cap X_{y_{k}}$, with $k \geq 1$, is equal to the set of states that are consistent with the measured output vectors and the initial state set $X_{0}$.

The computation of the exact uncertain state set is difficult. In practice, this set is approximated by conservative outer bounds to reduce the complexity. This paper presents a new method to compute an outer approximation using a zonotopebased procedure. Let us consider that an outer bound of the exact uncertain state set denoted $\hat{X}_{k-1}$ is available at time instant $k-1$. Suppose that a measured output vector $y_{k}$ is obtained at time instant $k$. Under these assumptions, an outer bound of the exact uncertain state set can be estimated using the following algorithm (similar to the Kalman filter which is based on a prediction step and an update step Brown \& Hwang (1997)).

\section{Algorithm 1}

Step 1. (Prediction) Given the system (1), compute a predicted state set $\bar{X}_{k}=A \hat{X}_{k-1} \oplus W$ bounding the uncertain trajectory.

Step 2. (Update) For $i=1, \ldots, n_{y}$ :

- Measurement: Compute the consistent state set $X_{y_{k / i}}$ using the output measurement $y_{k / i}$;

- Correction: Compute an outer approximation $\hat{X}_{k / i}$ of the intersection between $X_{y_{k / i}}$ and $\hat{X}_{k / i-1}$, with $\hat{X}_{k / 0}=\bar{X}_{k}$.

The guaranteed state estimation obtained at time instant $k$ is $\hat{X}_{k / n_{y}}$. This algorithm will be detailed in the next section.

\section{Main results}

At each time, the system (1) has $n_{y}$ available measurements, i.e., strips represented by $y_{k / i}=c_{i}^{\top} x_{k}+v_{k / i}, i=1, \ldots, n_{y}$, with $c_{i} \in \mathbb{R}^{n_{x}}$. Here $c_{i}^{\top}$ is the $i$-th row of matrix $C$ and the noise $v_{k / i}$ is bounded by the interval $V_{i}=\phi_{i} \mathbf{B}^{1}$, with $\phi_{i}=\Phi_{i i}$.

Supposing an outer approximation of the state set $\hat{X}_{k-1}=$ $\hat{p}_{k-1} \oplus \hat{H}_{k-1} \mathbf{B}^{r}$ at the time instant $k-1$, then the predicted state set at the next instant $\bar{X}_{k}$ can be computed as follows:

$$
\bar{X}_{k}=A \hat{p}_{k-1} \oplus\left[A \hat{H}_{k-1} \quad F\right] \mathbf{B}^{r+n_{\omega}}
$$

The exact estimation set at time instant $k$ will be obtained after intersecting the predicted state set with the consistent state set given by the measured output vector $y_{k}$. In the general case, an outer approximation of this set can be found by intersecting the predicted zonotopic state set with the first measurement strip, using Property 6. Then this intersection is outer approximated by a new zonotope which is further intersected with the second measurement strip. The procedure is repeated until the last measurement strip (i.e., for $1 \leq i \leq n_{y}$ ), leading to:

$$
\hat{X}_{k / i}\left(\lambda_{1}, \ldots, \lambda_{i}\right)=\hat{p}_{k / i}\left(\lambda_{1}, \ldots, \lambda_{i}\right) \oplus \hat{H}_{k / i}\left(\lambda_{1}, \ldots, \lambda_{i}\right) \mathbf{B}^{r+n_{\omega}+i}
$$

with $\hat{p}_{k / i}\left(\lambda_{1}, \ldots, \lambda_{i}\right)=\hat{p}_{k / i-1}\left(\lambda_{1}, \ldots, \lambda_{i-1}\right)+\lambda_{i}\left(y_{k / i}-\right.$ $\left.c_{i}^{\top} \hat{p}_{k / i-1}\left(\lambda_{1}, \ldots, \lambda_{i-1}\right)\right), \quad \hat{p}_{k / 0}=A \hat{p}_{k-1}, \hat{H}_{k / i}\left(\lambda_{1}, \ldots, \lambda_{i}\right)=$ $\left[\kappa_{i} \hat{H}_{k / i-1}\left(\lambda_{1}, \ldots, \lambda_{i-1}\right) \quad \phi_{i} \lambda_{i}\right], \kappa_{i}=I-\lambda_{i} c_{i}^{\top}, \hat{H}_{k / 0}=\left[\begin{array}{ll}A \hat{H}_{k-1} & F\end{array}\right]$.

The zonotopic guaranteed state estimation set at instant $k$ is denoted by $\hat{X}_{k}=\hat{X}_{k / n_{y}}$. This procedure is illustrated in Fig. 1 for a two-output system. At time instant $k$ the predicted state set is represented by the zonotope $\bar{X}_{k}$. Firstly, this set is intersected with the strip obtained by the first element of the measured output $\left|c_{1}^{\top} x-y_{k / 1}\right| \leq \phi_{1}$. Secondly, this intersection is approximated by the zonotope $\hat{X}_{k / 1}$ (dash line) using Property 6 . The procedure is repeated with $\hat{X}_{k / 1}$ and the strip obtained by the second element of the measured output $\left|c_{2}^{\top} x-y_{k / 2}\right| \leq \phi_{2}$, leading to the outer approximation $\hat{X}_{k / 2}$ of this intersection. The guaranteed state estimation set is then the zonotope $\hat{X}_{k / 2}$ (dash-dot line). Note that the order of the considered measurement strips can influence the accuracy of the estimation.

To obtain the guaranteed state estimation, the free vectors $\lambda_{i}$, with $i=1, \ldots, n_{y}$, must be computed. In the next part, two procedures are proposed to compute these vectors.

\subsection{First approach}

The vectors $\lambda_{1}, \ldots, \lambda_{n_{y}}$ are computed by considering $n_{y}$ separate single-output systems. The computation of $\lambda_{1}$ is detailed in the following and the computation of the vectors $\lambda_{2}, \ldots, \lambda_{n_{y}}$ 


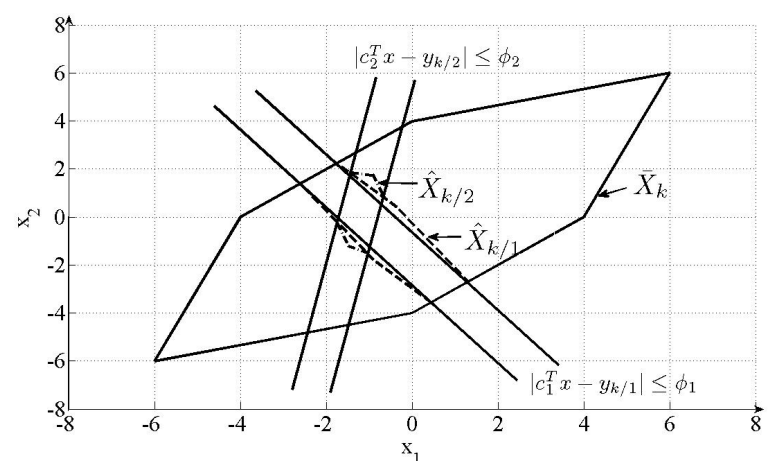

Figure 1: State estimation for Multi-Output systems

is similar. Consider the system (1) with the first component of the output measurement $y_{k / 1}$ and the constant known matrix $A$ :

$\left\{\begin{array}{l}x_{k+1}=A x_{k}+\omega_{k} \\ y_{k / 1}=c_{1}^{\top} x_{k}+v_{k / 1}\end{array}\right.$

Suppose the guaranteed state estimation at time instant $k-1$ is the zonotope $\hat{X}_{k-1}$ and its $P$-radius is $L_{k-1}$. Thus, the guaranteed state estimation at time instant $k$ is obtained similar to (3). The main idea consists in computing a matrix $P=P^{\top}>0$ and a vector $\lambda_{1}$ such that at each sample time the $P$-radius of the zonotopic state estimation set (i.e., $L_{k}$ ) and, hence, the zonotopic state estimation set are not increased. The non-increasing condition on the $P$-radius can be expressed in a mathematical formulation as follows. The decrease of the $P$-radius (i.e., $L_{k}$ ) is ensured by the expression $L_{k} \leq \beta L_{k-1}$, with $\beta \in(0,1]$. Due to the presence of state perturbations and measurement noise, this condition is difficult to verify. A relaxation of this condition can be $L_{k} \leq \beta L_{k-1}+\epsilon$, with $\epsilon$ a positive constant which permits to bound the influence of perturbations and measurement noises. For $\epsilon=\max _{\gamma \in \mathbf{B}^{n_{\omega}}}\|F \gamma\|_{2}^{2}+\phi_{1}^{2}>0$, this leads to:

$$
L_{k} \leq \beta L_{k-1}+\max _{\gamma \in \mathbf{B}^{n_{\omega}}}\|F \gamma\|_{2}^{2}+\phi_{1}^{2}, \text { with } \beta \in(0,1]
$$

or in an equivalent form:

$$
\max _{\hat{z} \in \mathbf{B}^{r+n_{\omega}+1}}\left\|\hat{H}_{k}\left(\lambda_{1}\right) \hat{z}\right\|_{P}^{2} \leq \max _{z \in \mathbf{B}^{r}} \beta\left\|\hat{H}_{k-1} z\right\|_{P}^{2}+\max _{\gamma \in \mathbf{B}^{n_{\omega}}}\|F \gamma\|_{2}^{2}+\phi_{1}^{2}
$$

with $\hat{z}=\left[\begin{array}{lll}z & \gamma & \eta\end{array}\right]^{\top} \in \mathbf{B}^{r+n_{\omega}+1}, z \in \mathbf{B}^{r}, \gamma \in \mathbf{B}^{n_{\omega}}, \eta \in \mathbf{B}^{1}$, and $\beta$ $\in(0,1]$. In addition, the next inequality is a sufficient condition of the expression (5):

$\max _{\hat{z} \in \mathbf{B}^{r+n_{\omega}+1}}\left(\left\|\hat{H}_{k}\left(\lambda_{1}\right) \hat{z}\right\|_{P}^{2}-\beta\left\|\hat{H}_{k-1} z\right\|_{P}^{2}-\|F \gamma\|_{2}^{2}-\phi_{1}^{2}\right) \leq 0$.

For all $\hat{z}, z, \gamma$, this inequality is implied by the following:

$$
\hat{z}^{\top} \hat{H}_{k}^{\top}\left(\lambda_{1}\right) P \hat{H}_{k}\left(\lambda_{1}\right) \hat{z}-\beta z^{\top} \hat{H}_{k-1}^{\top} P \hat{H}_{k-1} z-\gamma^{\top} F^{\top} F \gamma-\phi_{1}^{2} \leq 0
$$

Because $\eta \in \mathbf{B}^{1}$, i.e., $\|\eta\| \leq 1$, the following expression is obtained $\phi_{1}^{2}\left(1-\eta^{2}\right) \geq 0$. Adding this term to the left-side of (6) leads to the following sufficient condition for (6):

$$
\begin{array}{r}
\hat{z}^{\top} \hat{H}_{k}^{\top}\left(\lambda_{1}\right) P \hat{H}_{k}\left(\lambda_{1}\right) \hat{z}-\beta z^{\top} \hat{H}_{k-1}^{\top} P \hat{H}_{k-1} z-\gamma^{\top} F^{\top} F \gamma-\phi_{1}^{2}+ \\
+\phi_{1}^{2}\left(1-\eta^{2}\right) \leq 0, \quad \forall \hat{z}, z, \gamma
\end{array}
$$

Multiplying the expression of $\hat{H}_{k}$ in (3) with the explicit form of $\hat{z}$ leads to $\hat{H}_{k}\left(\lambda_{1}\right) \hat{z}=\left(I-\lambda_{1} c_{1}^{\top}\right) A \hat{H}_{k-1} z+F \gamma+\phi_{1} \lambda_{1} \eta=$ $\kappa_{1} A \hat{H}_{k-1} z+F \gamma+\phi_{1} \lambda_{1} \eta$. Denote $\theta=\hat{H}_{k-1} z$, then the inequality (7) can be written in the matrix formulation:

$$
\left[\begin{array}{l}
\theta \\
\gamma \\
\eta
\end{array}\right]^{\top} \underbrace{\left[\begin{array}{ccc}
A_{11} & A_{12} & A_{13} \\
* & A_{22} & A_{23} \\
* & * & A_{33}
\end{array}\right]}_{J}\left[\begin{array}{l}
\theta \\
\gamma \\
\eta
\end{array}\right] \leq 0, \quad \forall \theta, \gamma, \eta
$$

with ' $*$ ' denoting the terms required for the symmetry of the matrix and $A_{11}=A^{\top} \kappa_{1}^{\top} P \kappa_{1} A-\beta P, A_{12}=A^{\top} \kappa_{1}^{\top} P \kappa_{1} F, A_{13}=$ $A^{\top} \kappa_{1}^{\top} P \phi_{1} \lambda_{1}, A_{22}=F^{\top} \kappa_{1}^{\top} P \kappa_{1} F-F^{\top} F, A_{23}=F^{\top} \kappa_{1}^{\top} P \phi_{1} \lambda_{1}$, and $A_{33}=\phi_{1}^{2} \lambda_{1}^{\top} P \lambda_{1}-\phi_{1}^{2}$. Using the definition of positive definite matrix allows to rewrite (8) as $J \leq 0, \forall \theta, \gamma, \eta \neq 0$. Using the explicit notations of $J$ and doing some manipulations, a matrix inequality is derived as:

$\left[\begin{array}{ccc}\beta P & 0 & 0 \\ 0 & F^{\top} F & 0 \\ 0 & 0 & \phi_{1}^{2}\end{array}\right]-\Xi P^{-1} \Xi^{\top} \geq 0$, with $\Xi=\left[\begin{array}{l}A^{\top} \kappa_{1}^{\top} P \\ F^{\top} \kappa_{1}^{\top} P \\ \lambda_{1}^{\top} P \phi_{1}\end{array}\right]$.

Using the Schur complement (Boyd et al. (1994)), this expression is equivalent to the following matrix inequality: $\left[\begin{array}{cccc}\beta P & 0 & 0 & A^{\top} P-A^{\top} c_{1} Y^{\top} \\ * & F^{\top} F & 0 & F^{\top} P-F^{\top} c_{1} Y^{\top} \\ * & * & \phi_{1}^{2} & Y^{\top} \phi_{1} \\ * & * & * & P\end{array}\right] \geq 0$

with the change of variable $Y=P \lambda_{1}$.

To verify (8) for $\forall A \in[A]$, with $[A]$ a convex set, we need to verify this inequality on each vertex $G_{i}$ of $[A]$, with $i=1, \ldots, 2^{q}$ and $q$ the number of interval elements of matrix $[A]$.

As the 2-norm is a convex function and $W$ is a convex set, the constant term $\psi=\max _{\gamma \in \mathbf{B}^{n_{\omega}}}\|F \gamma\|_{2}^{2}$, where $\gamma \in \mathbf{B}^{n_{\omega}}$, can be easily computed. Then the condition (4) can be written as $L_{k} \leq \beta L_{k-1}+\psi+\phi_{1}^{2}$. At infinity, this expression is equivalent to $L_{\infty}=\beta L_{\infty}+\psi+\phi_{1}^{2}$, leading to $L_{\infty}=\frac{\phi_{1}^{2}+\psi}{1-\beta}$, with the additional hypothesis on the convergence of the $\left\{L_{k}\right\}$ sequence. Let us consider an ellipsoid $E=\left\{x: x^{\top} P x \leq \frac{\phi_{1}^{2}+\psi}{1-\beta}\right\}$ which can be normalized to $E=\left\{x: x^{\top} \frac{(1-\beta) P}{\phi_{1}^{2}+\psi} x \leq 1\right\}$. To minimize the $P$-radius (i.e., $L_{\infty}$ ) of the zonotope, the ellipsoid of the smallest diameter must be found (Boyd et al. (1994)). This leads to solving the following eigenvalue problem (EVP), i.e., to finding the values of $P=P^{\top}>0, P \in \mathbb{R}^{n_{x} \times n_{x}}$ and $\lambda_{1} \in \mathbb{R}^{n_{x}}$ :

$$
\begin{aligned}
& \max _{\tau, \beta, P} \tau \\
& \text { subject to } \frac{(1-\beta) P}{\phi_{1}^{2}+\psi} \geq \tau I_{n_{x}}
\end{aligned}
$$

with $\tau \in \mathbb{R}^{+}, \beta \in(0,1]$ and the identity matrix $I_{n_{x}} \in \mathbb{R}^{n_{x} \times n_{x}}$. Then, the diameter of the obtained ellipsoid is given by $\frac{2}{\sqrt{\tau^{*}}}$ (Boyd et al. (1994)), with $\tau^{*}$ the optimal value of $\tau$.

Finally, to find the values of $P=P^{T}>0, P \in \mathbb{R}^{n_{x} \times n_{x}}$ and 
$\lambda_{1} \in \mathbb{R}^{n_{x}}$, the following optimization problem must be solved:

$$
\begin{aligned}
& \max _{\tau, \beta, P, Y} \tau \\
& \text { subject to } \tau>0, \\
& {\left[\begin{array}{cccc}
\beta P & 0 & 0 & G_{i}^{\top} P-G_{i}^{\top} c_{1} Y^{\top} \\
* & F^{\top} F & 0 & F^{\top} P-F^{\top} c_{1} Y^{\top} \\
* & * & \phi_{1}^{2} & Y^{\top} \phi_{1} \\
* & * & * & P
\end{array}\right] \geq 0}
\end{aligned}
$$

where $G_{i}$ are the vertices of the interval matrix $[A]$, with $i=$ $1, \ldots, 2^{q}$. As $\beta$ is a scalar variable, this optimization problem can be efficiently solved by using a BMI (Bilinear Matrix Inequality) solver (e.g., Penbmi Kocvara \& Stingl (2003)) or by executing a simple search-loop on $\beta$. In the optimization problem (9), the decision variables are: $P=P^{\top} \in \mathbb{R}^{n_{x} \times n_{x}}, Y \in \mathbb{R}^{n_{x}}$, $\beta \in(0,1]$ and $\tau \in \mathbb{R}^{+}$. Thus, the total number of the scalar decision variables is $\frac{n_{x}\left(n_{x}+1\right)}{2}+n_{x}+2$. The dimensions of inequalities (9) are $1, n_{x} \times n_{x},\left(2 n_{x}+n_{\omega}+1\right) \times\left(2 n_{x}+n_{\omega}+1\right)$, resp.

When $A$ is unknown but belongs to the interval matrix $[A]$, the predicted state set $\bar{X}_{k}$ can not be directly computed by the expression (2) at each iteration. This set is replaced by the following outer approximation. The starting point is given by equation (2). As $A$ is bounded by the interval matrix [A], an outer approximation of $\bar{X}_{k}$ can be obtained by $[A] \hat{p}_{k-1} \oplus$

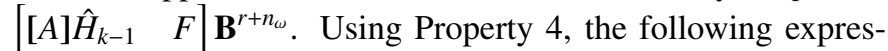
sion is true $[A] \hat{p}_{k-1} \in(\operatorname{mid}[A]) \hat{p}_{k-1} \oplus \operatorname{rs}\left((\operatorname{rad}[A])\left|\hat{p}_{k-1}\right|\right) \mathbf{B}^{n_{x}}$. In addition, Properties 4 and 5 imply that $[A] \hat{H}_{k-1} \mathbf{B}^{r} \subseteq$ $\left[(\operatorname{mid}[A]) \hat{H}_{k-1} \quad r s\left((\operatorname{rad}[A])\left|\hat{H}_{k-1}\right|\right)\right] \mathbf{B}^{r+n_{x}}$.

The Minkowski sum of the last two expressions leads to $[A] \hat{p}_{k-1} \oplus[A] \hat{H}_{k-1} \mathbf{B}^{r} \subseteq(\operatorname{mid}[A]) \hat{p}_{k-1} \oplus \operatorname{rs}\left((\operatorname{rad}[A])\left|\hat{p}_{k-1}\right|\right) \mathbf{B}^{n} \oplus$ $\left.\oplus\left[(\operatorname{mid}[A]) \hat{H}_{k-1} \quad r s\left((\operatorname{rad}[A])\left|\hat{H}_{k-1}\right|\right)\right)\right] \mathbf{B}^{r+n}$.

Therefore, the zonotope representing the outer approximation of $\bar{X}_{k}$ is $(\operatorname{mid}[A]) p_{k-1} \oplus Q \mathbf{B}^{l}$, with $l=r+2 n_{x}+n_{\omega}$ and $Q=$ $\left[(\operatorname{mid}[A]) \hat{H}_{k-1} \quad r s\left((\operatorname{rad}[A])\left|\hat{H}_{k-1}\right|\right) \quad r s\left((\operatorname{rad}[A])\left|\hat{p}_{k-1}\right|\right) \quad F\right]$. This zonotope is formed by generators which depend on $\hat{H}_{k-1}$ and $\hat{p}_{k-1}$. From the quadratic stability assumption on $A \in[A]$ matrix and considering that $\operatorname{rad}[A]$ is small enough, these allow to bound the effect of the considered interval uncertainties (i.e., the generator $(\operatorname{rad}[A])\left|\hat{p}_{k-1}\right|$ is bounded) and, thus, the states of the system converge to a region containing the origin. Moreover, the computation of vector $\lambda$ depends only on the vertices of the interval matrix $[A]$ and not $\bar{X}_{k}$ and this outer approximation is done at each time instant. This implies that the approximation has a limited effect on the $P$-radius of the obtained state outer bound. Moreover, since the $P$-radius has a shrinking nature when $\operatorname{rad}[A]$ is zero, it follows that for small enough values of $\operatorname{rad}[A]$ the $P$-radius will also be shrinking with each iteration of the algorithm.

\subsection{Second Approach}

As the coupling effect of multi-output systems is not considered, the first proposed method can be conservative. To reduce the conservatism of the previous method, the following procedure is proposed. Using $y_{k / 1}$, the predicted state set $\bar{X}_{k}$, expressions (3) and (9) allow us to compute $\lambda_{1}$ and a smaller zonotope
$\hat{X}_{k / 1}$. Intersecting this new zonotope with the strip corresponding to $y_{k / 2}$ (supposing $\lambda_{1}$ known from the previous step) leads to $\lambda_{2}$ and another zonotope $\hat{X}_{k / 2}$. This procedure is repeated until the last component of the output vector $y_{k / n_{y}}$ (supposing all the previous vectors $\lambda_{1}, \ldots, \lambda_{n_{y}-1}$ to be known). The following algorithm describes this off-line procedure.

\section{Algorithm 2}

Step 1 . Using the measurement $y_{k / 1}$ and (9), compute $\lambda_{1}$. For $j=2, \ldots, n_{y}$ :

Step $j$ : Using the measurement information of $y_{j}$ and the previous vectors $\lambda_{1}, \ldots, \lambda_{j-1}$, compute $\lambda_{j}$.

The guaranteed state estimation set at Step $j$ is computed by replacing $i$ by $j$ in the expression (3). A similar condition on the $P$-radius of the zonotopic estimation set is applied, leading to $\max _{z \in \mathbf{B}^{r+n_{\omega}+j}}\left\|\hat{H}_{k / j} \hat{z}\right\|_{P}^{2} \leq \max _{z \in \mathbf{B}^{r}} \beta\left\|\hat{H}_{k-1} z\right\|_{P}^{2}+\max _{\gamma \in \mathbf{B}^{n_{\omega}}}\|F \gamma\|_{2}^{2}+\phi_{1}^{2}+\ldots+\phi_{j}^{2}$, with $\hat{z}=\left[\begin{array}{lllll}z^{\top} & \gamma^{\top} & \eta_{1} & \ldots & \eta_{j}\end{array}\right]^{\top} \in \mathbf{B}^{r+n_{\omega}+j}, z \in \mathbf{B}^{r}, \gamma \in \mathbf{B}^{n_{\omega}}$, $\eta_{j} \in \mathbf{B}^{1}$, and $\beta \in(0,1]$.

At Step $j$, similar to the first approach the following optimization problem is obtained for $i=1, \ldots, 2^{q}$ :

$$
\begin{aligned}
& \max _{\tau, \beta, P, Y} \tau \\
& \text { subject to } \tau>0, \frac{(1-\beta) P}{\psi+\phi_{1}^{2}+\phi_{2}^{2}+\ldots+\phi_{j}^{2}} \geq \tau I_{n_{x}} \\
& {\left[\begin{array}{ccccccc}
\beta P & 0 & 0 & \cdots & 0 & 0 & \left(\left(\prod_{l=1}^{j} \kappa_{j+1-l}\right) G_{i}\right)^{\top} P \\
* & F^{\top} F & 0 & \cdots & 0 & 0 & \left(\left(\prod_{\substack{j=1 \\
j}}^{j-1} \kappa_{j+1-l}\right) F\right)^{\top} P \\
* & * & \phi_{1}^{2} & \cdots & 0 & 0 & \left(\left(\prod_{l=1}^{j-1} \kappa_{j+1-l}\right) \phi_{1} \lambda_{1}\right)^{\top} P \\
\vdots & \vdots & \vdots & \ddots & \vdots & \vdots & \vdots \\
* & * & * & \cdots & \phi_{j-1}^{2} & 0 & \left(\kappa_{j} \phi_{j-1} \lambda_{j-1}\right)^{\top} P \\
* & * & * & \cdots & * & \phi_{j}^{2} & \phi_{j} Y^{\top} \\
* & * & * & \cdots & * & * & P
\end{array}\right] \geq 0}
\end{aligned}
$$

with $G_{i}$ the vertices of the interval matrix $[A], q$ the number of interval elements of $[A]$ and the decision variables $Y=P \lambda_{j}$, $P=P^{\top}>0, \tau \in \mathbb{R}^{+}$and $\beta \in(0,1]$.

The order used to take into account the different measurements can influence on the precision of the estimation (the size of the guaranteed state estimation), thus, to obtain the best performance, $n_{y}$ ! combinations of the order can be tried.

\section{Illustrative example}

The example considered here is inspired from (Alamo et al. (2005)) in order to compare the performance of the proposed algorithm to the existing approaches:

$$
\left\{\begin{array}{l}
x_{k+1}=\left[\begin{array}{cc}
0 & -0.5 \\
1 & 1+0.3 \delta
\end{array}\right] x_{k}+0.02\left[\begin{array}{c}
-6 \\
1
\end{array}\right] \omega_{k} \\
y_{k}=\left[\begin{array}{cc}
-2 & 1 \\
1 & 1
\end{array}\right] x_{k}+\left[\begin{array}{cc}
0.2 & 0 \\
0 & 0.2
\end{array}\right] v_{k}
\end{array}\right.
$$

with parameter uncertainty $|\delta| \leq 1$, measurement noise $\left\|v_{k}\right\|_{\infty} \leq$ 1 and state perturbation $\left\|\omega_{k}\right\|_{\infty} \leq 1$. The values of $\delta, v_{k}$, $\omega_{k}$ are generated by random functions of Matlab ${ }^{\circledR}$. The initial state belongs to the box $3 B^{2}$ and is randomly generated. The order of the $m$-zonotopes is limited to $m \leq 20$ for the 


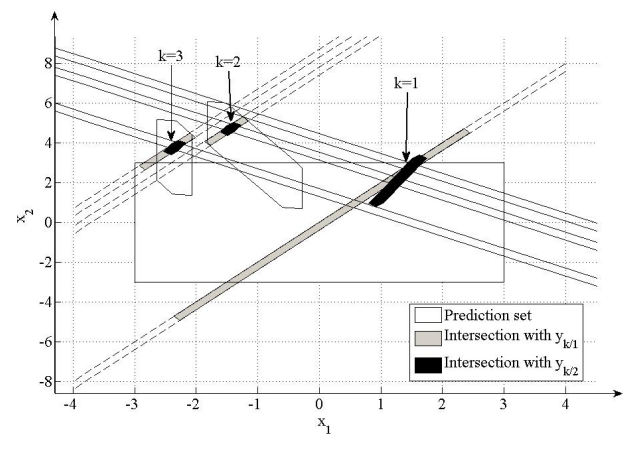

Figure 2: Intersection $\hat{X}_{k}$ between $\bar{X}_{k}$ and $X_{y_{k}}$

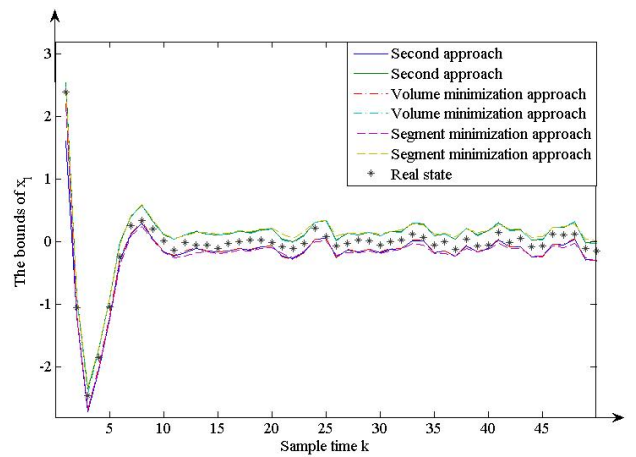

Figure 3: Guaranteed bound of $x_{1}$

purpose of a fast simulation. In this example, the results obtained by the first approach (subsection 4.1) and the second approach (subsection 4.2) are compared with the results obtained by the segment minimization approach and the volume minimization approach from Alamo et al. (2005) applied for the multivariable case. The first approach gives the correction factors $\lambda_{1}=\left[\begin{array}{ll}-0.2137 & 0.5726\end{array}\right]^{\top}$ and $\lambda_{2}=\left[\begin{array}{lll}0.3684 & 0.3570\end{array}\right]^{\top}$. The correction factors computed by the second approach are $\lambda_{1}=\left[\begin{array}{lll}-0.2137 & 0.5726\end{array}\right]^{\top}$ and $\lambda_{2}=\left[\begin{array}{lll}0.2839 & 0.5085\end{array}\right]^{\top}$. The simulation results are shown in Figures 2, 3, 4, 5, 6.

Figure 2 shows the evolution of the predicted state set and the outer approximation of the exact uncertain state set at time

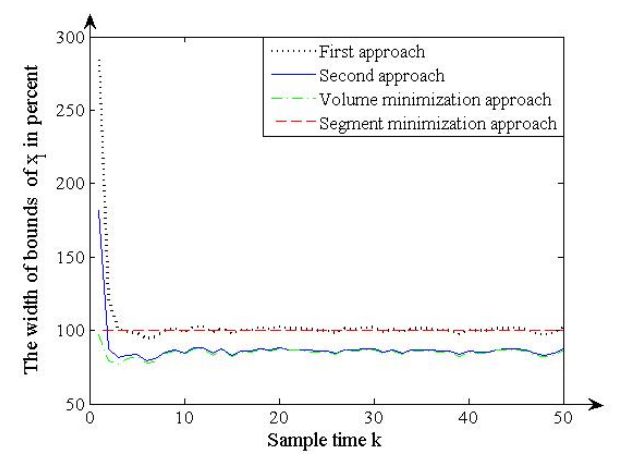

Figure 4: Comparison of the bound's width of $x_{1}$

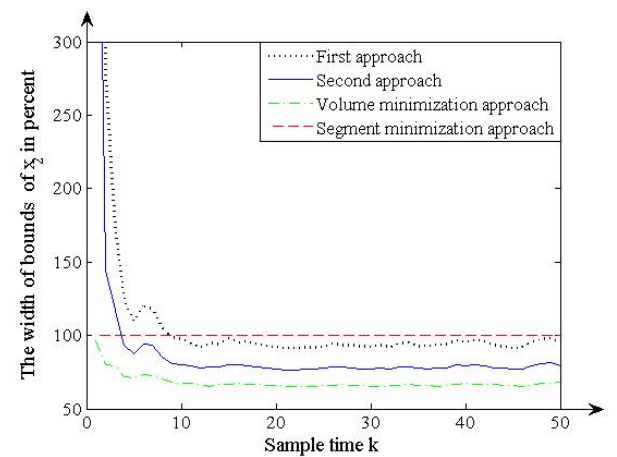

Figure 5: Comparison of the bound's width of $x_{2}$

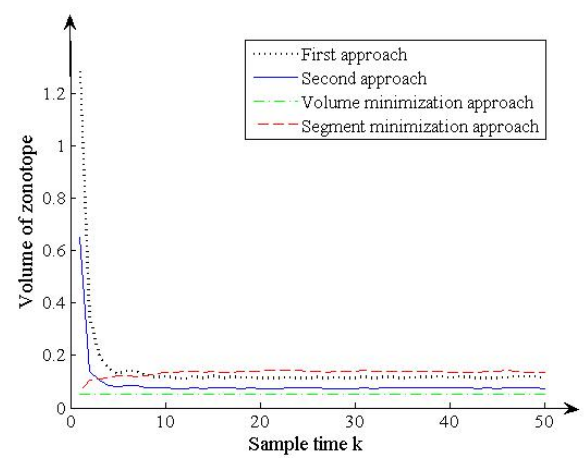

Figure 6: Comparison of the volume of state estimation zonotopic set

instant $k=1,2,3$ using the second proposed approach. The intersection between the predicted state set and the strip obtained from $y_{k / 1}$ (dash line) is approximated by a zonotope (grey), then this zonotope is intersected with the strip obtained from $y_{k / 2}$. Finally, the guaranteed state estimation at time instant $k$ (black) is the outer approximation (which is rapidly reduced at each iteration due to condition (5)) of this intersection.

Figures 3, 4, 5, 6 compare the obtained bound on $x_{k / 1}, x_{k / 2}$ and the volume of the guaranteed bound of the state obtained with different methods: the segment minimization method (Alamo et al. (2005)), the volume minimization method (Alamo et al. (2005)), and the proposed $P$-radius minimization methods. In Figure 3, the real system states are found between the upper bound and the lower bound of $x_{k / 1}$, which confirms that these bounds are well estimated. As the bounds obtained by different methods are similar, the Figures 4 and 5 compare the width of the bounds for $x_{k / 1}$ and $x_{k / 2}$ computed by different methods, considering the segment minimization algorithm as reference. The bound on $x_{k / 1}, x_{k / 2}$ and the volume of the zonotope obtained by the proposed methods are smaller than the one obtained by the segment minimization method. The accuracy is almost the same in the second proposed method and the volume minimization method.

The results obtained by the first approach and the second approach are also compared. We can see the accuracy of the second approach is better than the one of the first approach which confirms a less conservative result of the second approach. 
Table 1: Total computation time after 50 samples

\begin{tabular}{lc} 
Algorithm & Time (second) \\
\hline Segment minimization & 0.0780 \\
First approach and second approach (without off-line BMI) & 0.0780 \\
First approach and second approach (off-line BMI included) & 1.2636 \\
Volume minimization & 22.2457 \\
\hline
\end{tabular}

Table 1 compares the computation time of different methods. These results are obtained with an Intel Core 2 Duo E8500 $3.16 \mathrm{GHz}$. The BMI optimization is solved with Penbmi solver, the volume minimization problem is dealt with the fmincon function of Matlab ${ }^{\circledR}$ and the segment minimization problem is solved with a simple computation. The online computation time is the same in the proposed methods and the segment minimization method. The computation time of the proposed methods is 20 times faster than the one of the volume minimization method. This can be explained by the fact that in the volume minimization method an optimization problem must be solved online at each sample time but in the proposed methods almost all the computation is dealt off-line.

To conclude, the proposed methods combine the lowcomplexity of the segment minimization and the good accuracy of the volume minimization.

\section{Conclusion}

A new approach based on the $P$-radius minimization allows the guaranteed state estimation for stable multi-output systems with bounded state perturbations and bounded noises. The procedure computes a zonotopic set of all the possible states that are consistent with the measured output vector and the given noise. The size of this zonotope is non-increasing at each sample time leading to a better estimation accuracy. Using the $P$ radius minimization offers a good trade-off between the complexity reflected by the computation time and the accuracy of the estimation. With the additional assumption on the quadratic stability of interval systems and based on bounded outer approximations, this approach still guarantees the convergence of the estimation in the presence of interval uncertainties.

Firstly, future works are related to the zonotopic guaranteed state estimation for uncertain multivariable systems. Considering all the measurements at the same time (i.e., all the vectors $\lambda_{1}, \ldots, \lambda_{n_{y}}$ must be computed at the same time) leads to a Polynomial Matrix Inequality (PMI). A sub-optimal solution for PMI problems can be found using different relaxation techniques (e.g., Henrion \& Lasserre (2011)); this can be investigated in the future. A more interesting and not trivial direction is to investigate: the consistent state set computed by all the strips of measurement at the same time (leading to a polytope), and then, the intersection of the obtained polytope with the zonotopic predicted state set. Secondly, further developments will focus on combining this estimation technique together with Tube-based Model Predictive Control. Finally, the proposed zonotopic set-membership estimation technique can be applied to fault detection and fault tolerant control purposes.

\section{References}

Alamo, T., Bravo, J. M., \& Camacho, E. F. (2005). Guaranteed state estimation by zonotopes. Automatica, 41, 1035-1043.

Alamo, T., Tempo, R., Ramírez, D. R., \& Camacho, E. F. (2008). A new vertex result for robustness problems with interval matrix uncertainty. Systems and Control Letters, 57, 474-481.

Althoff, M., Stursberg, O., \& Buss, M. (2007). Reachability analysis of linear systems with uncertain parameters and inputs. In Proc. of the 46th IEEE $C D C$ (pp. 726-732). volume 41. New Orleans, LA, USA.

Bertsekas, D. P., \& Rhodes, I. B. (1971). Recursive state estimation for a setmembership description of uncertainty. IEEE TAC, 16(2), 117-128.

Boyd, S., Ghaoui, L. E., Feron, E., \& Balakrishnan, V. (1994). Linear Matrix Inequalities in system and control theory. Philadelphia: SIAM.

Brown, R. G., \& Hwang, P. Y. C. (1997). Introduction to random signals and applied Kalman filtering. John Wiley and Sons.

Chernous'ko, F. L. (1994). State estimation for dynamic systems. CRC Press.

Combastel, C. (2003). A state bounding observer based on zonotopes. In Proc. of ECC. Cambridge, UK.

Combastel, C., Zhang, Q., \& Lalami, A. (2008). Fault diagnosis based on the enclosure of parameters estimated with an adaptive observer. In Proc. of the 17th World Congress IFAC (pp. 7314-7319). Seoul, Korea.

Durieu, C., Walter, E., \& Polyak, B. (2001). Multi-input multi-output ellipsoidal state bounding. JOTA, 111(2), 273-303.

Guibas, L. J., Nguyen, A., \& Zhang, L. (2005). Zonotopes as bounding volume. In Proc. of the Symposium on Discrete Algorithm (pp. 803-812).

Henrion, D., \& Lasserre, J. B. (2011). Inner approximations for polynomial matrix inequalities and robust stability regions. Technical Report LAASreport 11210.

Kalman, R. E. (1960). A new approach to linear filtering and prediction problems. Transactions of the ASME-Journal of Basic Engineering, 82, 35-45.

Kocvara, M., \& Stingl, S. (2003). PENNON - a code for convex nonlinear and semidefinite programming. Optimization Methods and Software, 18(3), 317-333.

Kühn, W. (1998). Rigorously computed orbits of dynamical systems without the wrapping effect. Computing, 61, 47-67.

Kurzhanski, A. B., \& Vályi, I. (1996). Ellipsoidal calculus for estimation and control. Birkhaüser Boston.

Le, V., Alamo, T., Camacho, E., Stoica, C., \& Dumur, D. (2011). A new approach for guaranteed state estimation by zonotopes. In Proc. of the 18th World Congress IFAC (pp. 9242-9247). Milan, Italy.

Le, V. T. H., Alamo, T., Camacho, E. F., Stoica, C., \& Dumur, D. (2012). Zonotopic set-membership estimation for interval dynamic systems. In Proc. of the 2012 ACC (pp. 6787-6792). Montréal, Canada.

Mao, W.-J., \& Chu, J. (2003). Quadratic stability and stabilization of dynamic interval systems. IEEE TAC, 48(6), 1007-1012.

Maybeck, P. S. (1979). Stochastic models, estimation and control. Academic Press.

Milanese, M., Norton, J., Piet-Lananier, S., \& Walter, E. (1996). Bounding approach to system identification. Plenum Press.

Plarre, K., \& Bullo, F. (2008). On Kalman filtering for detectable systems with intermittent observations. IEEE TAC, 54(2), 386-390.

Polyak, B. T., Nazin, S. A., Durieu, C., \& Walter, E. (2004). Ellipsoidal parameter or state estimation under model uncertainty. Automatica, 40, 1171-1179.

Puig, V., Cugueró, P., \& Quevedo, J. (2001). Worst-case estimation and simulation of uncertain discrete-time systems using zonotopes. In Proc. of ECC. Portugal.

Schweppe, F. C. (1968). Recursive state estimation: unknown but bounded errors and system inputs. IEEE TAC, 13(1), 22-28.

Sorenson, H. (1983). Special issue on applications of Kalman filtering. IEEE TAC, 28(3), 253-434.

Stoican, F., Olaru, S., Doná, J. D., \& Seron, M. (2011). Zonotopic ultimate bounds for linear systems with bounded disturbances. In Proc. of the 18th World Congress IFAC (pp. 9224-9229). Milan, Italy.

Vicino, A., \& Zappa, G. (1996). Sequential approximation of feasible parameter sets for identification with set membership uncertainty. IEEE TAC, 41, 774-785.

Walter, E., \& Piet-Lahanier, H. (1989). Exact recursive polyhedral description of the feasible parameter set for bounded-error models. IEEE TAC, 34(8), 911-915.

Witsenhausen, S. H. (1968). Sets of possible states of linear systems given perturbed observations. IEEE TAC, 13, 556-558. 